\title{
Integration and Evaluation of Peer Grading in a Graduate-level Engineering Design Course
}

\section{Elissa Morris, Texas A\&M University}

Elissa Morris is a PhD student at Texas A\&M University under the advising of Dr. Daniel A. McAdams. Her research interests include bioinspired design, origami-inspired design, and engineering education.

\section{Dr. Daniel A. McAdams, Texas A\&M University}

Dr. McAdams is an Associate Professor of Mechanical Engineering in the Department of Mechanical Engineering and Graduate Program Director at Texas A\&M University. He joined the department in January of 2008 after serving as an Associate (2005-2007) and Assistant Professor (1999-2005) of Mechanical Engineering at the Missouri University of Science and Technology. He received his Ph.D. from the University of Texas at Austin in 1999. He teaches undergraduate courses in design methods, biologically inspired design, and machine element design and graduate courses in product design and dynamics. Dr. McAdams research interests are in the area of design theory and methodology with specific focus on functional modeling; innovation in concept synthesis; biologically inspired design methods; inclusive design; and technology evolution as applied to product design. He has edited a book on biologically inspired design. 


\title{
Integration and evaluation of peer grading in a graduate-level engineering design course
}

\begin{abstract}
A peer grading method is developed and integrated into a graduate-level engineering product design course. The objective of the peer grading process is to improve the students' design skillset. Students form teams to work on a design project throughout the course, applying the methods discussed in class to their specific project. Each team submits a project report in phases throughout the semester. The first two phases of the report are peer graded by themselves and two other teams in the class. Teams also grade their graders to ensure accountability and increase grading fairness and high feedback quality. To eliminate potential bias, the entire grading process is blind. Any identifying information in the reports is removed prior to peer grading and a confidential labeling system is integrated for instructor use only. The teams provide cardinal and ordinal grades for each report. To guide the grading process, students are provided with a grading rubric and a detailed report outline which informs students of required report elements. Anonymous peer evaluation of each team member within each group is also required. Thirty-two students complete a survey regarding the peer grading experience at the end of the semester. The survey provides results that qualitatively measure the efficacy of the peer grading method. Qualitative analysis of the survey responses indicate the peer grading method successfully reinforces and improves understanding of engineering design concepts. Proposals for revision and improvement of the peer grading method based on the survey results are also discussed and remain as future work.
\end{abstract}

\section{Introduction}

According to Torrance et al., the practice and study of assessment in education has experienced three major transitions - 'assessment of learning' to 'assessment for learning' to 'assessment as learning' where assessment dominates learning experiences [1]. Considering the potential of assessment to significantly contribute to student learning, a peer grading method is integrated into a graduate-level engineering product design course. Peer grading is a well-studied pedagogical technique used in various ways depending on educational discipline and learning outcomes [2]. Peer grading provides numerous pedagogical benefits such as increasing motivation for learning course content [3,4], strengthening critical thinking skills [5], and improving the development of immediate and long-term learning aptitude $[6,7]$.

The primary motivation for integrating peer grading in an advanced product design course is to increase the student's understanding of engineering design concepts and to promote design thinking. Various methods exist in the literature that are effective for teaching design concepts [8]. However, the efficacy of peer grading as an instructional technique for engineering design is not well-studied. Researchers integrate peer assessment methods in engineering design courses but students only assess themselves and their team members $[9,10]$. However, the authors of this present work use peer grading for internal team assessment and assessment of other teams in the course, which allows students to compare their design process to others. The literature also presents cases where peer assessment is used in engineering courses unrelated to product design 
[11-14]. However, the authors are specifically interested in the use of peer grading to teach engineering design concepts and to develop the students' design skillset.

The secondary motivation for implementing peer grading is to reduce the time and effort required by the professor for grading. Assignments from project-based courses typically require more time and effort to grade due to the open-ended nature of the work [15]. Integrating peer grading requires an initial time investment on behalf of the professor to create grading rubrics, prepare peer grading training sessions, and develop other organizational methods to ensure successful implementation. Despite this initial time investment, peer grading can significantly reduce the time and effort required by the professor to grade students' work [2].

The accuracy of peer grading is debatable. Research shows that students tend to grade liberally compared to professional graders, especially on material that tests higher level cognitive skills [16]. Conversely, researchers have demonstrated that peer grading and professional grading yield similar results [17]. Accuracy of peer grading varies based on factors such as education level, discipline, material being graded, and grading rubric clarity. Feedback quality is another debatable issue regarding peer grading methods. However, research shows that providing accountability to graders though 'grading the grader' procedures increases feedback quality [18]. Therefore, grading the grader is an element of the peer grading procedure presented here.

The purpose of this paper is to present a peer grading methodology for engineering design courses and an assessment of the methodology through qualitative analysis. The paper is structured accordingly: Section 2 describes the peer grading procedure as used in an advanced engineering product design course, Section 3 presents the qualitative analysis of survey responses regarding the efficacy of the peer grading method, Section 4 includes a discussion of the qualitative analysis results and open-ended survey responses, and Section 5 presents suggested areas for future work and conclusions.

\section{Peer grading methodology in advanced product design course}

A peer grading method is used in a graduate-level product design course offered in the mechanical engineering curriculum. The course objectives and procedures for peer grading integration are discussed in the following sub-sections.

\subsection{Course objectives and design project requirements}

The course focuses on systematic design methodology. Course topics include customer needs analysis, functional modeling, idea generation, and decision making. The fundamental course objective is to equip students with skills necessary for success as a design engineer or design researcher. Completion of a semester-long project is required which provides students the opportunity to apply learned design techniques to product design. The class consists of 45 students, which allows for 13 design teams with 3 to 4 members on each team. Each team selects a unique design project and must complete a technical report explaining their design process for the project. The report is divided into three phases, or deliverables, which must be completed by specified dates throughout the semester. The design techniques discussed in lecture must be 
included in the report as part of each team's design process. Therefore, report deliverables align with lecture topics covered.

\subsection{Peer grading procedure}

The peer grading method is used to assess the first two phases of the report, while the professor grades the final phase. Each team grades a total of three reports in each phase, including their own report and two anonymous reports from other teams in the class. The two anonymous reports are randomly assigned to each team by teaching assistants. The teaching assistants delete any identifying information prior to distributing reports to teams for peer grading to ensure anonymity. The teaching assistants develop a confidential numbering system to label the reports prior to distribution for peer grading.

Peer grading of the design reports must be completed as a team. Instructions and a grading rubric are provided to the student teams to guide the peer grading process, shown in Figure 1. Also, a detailed report outline informs students what must be included within each section of the report to further guide their grading. Each team assigns an overall cardinal score (4 to 0 with 4 being the highest score) for each report. Students calculate the average of all section scores to inform their judgment when determining the overall cardinal score. Students must also assign an ordinal rank (best to worst) to grade the reports. The ordinal ranking distinguishes the highest quality report in the event that identical cardinal scores are given for multiple reports. Students must also provide constructive feedback when grading to justify the score they assign. 


\section{Peer Grading Instructions}

This is a team homework. Your task is to grade each report in your assignment queue. Note, one is your report and the other two reports are from other teams in the class. Each report will have a cardinal score assigned by you as a team $-4,3,2,1$, or 0 (A, B, C, D, or F). Also, provide ordinal rankings for the reports from best to worst. Your cardinal ratings need to track with your ordinal rankings. If not, fix it.

Best report ; cardinal score

Second best report ; cardinal score

Third best report ; cardinal score

\section{Cardinal Score Rubric}

4 = The design team did a fantastic job. I (as a grader) understand everything and have no questions. I understand how to determine success or failure for a resulting design.

3 = The design team did a solid job. I (as a grader) more or less understand most of it. I'm a little bit unclear, but could probably successfully design the solution.

2 = The design team did a somewhat sloppy job. I (as a grader) am not sure exactly what the problem is and how to determine if a successful design is achieved. I'd like access to the design team and customers to ask some questions.

1 = The design team did a very poor job overall. This document looks like it was stapled together from separate parts and the parts haven't been integrated. The design problem isn't clear. Multiple steps aren't done or look "faked."

$0=N / A$

Grade each section of the report using the cardinal scale.

a) Average of all section grades combined.

b) Your overall score for the report. The total score need not be the average of each section. The total score is a bit more of a high level assessment and depends on your judgment.

\section{Submitting Feedback}

Provide significant feedback to the team about what is good and not good about the report. For example, you can print out the report and write comments, or use some inline/overline commenting in PDF or Word format. Regardless, you must provide thorough feedback to the team you are grading. Submit the graded work electronically. If you use hand-written notes on the report, scan it in to submit it at a high enough resolution so that others can see your feedback.

Figure 1 - Instructions for the peer grading process

The primary objective of implementing the peer grading method is to reinforce design concepts taught in lecture and to further develop the students' design skillset. Peer grading is implemented to expose students to various examples of design, to provide further opportunities for teamwork, and to facilitate reflective practice. The peer grading method is also utilized to motivate students to produce higher quality work considering their peers are evaluating them.

\subsection{Grade the grader procedure}

After the teams complete peer grading, the graded reports are returned to the appropriate teams using the confidential numbering system developed by the teaching assistants. Each team grades their graders based on the quality of feedback provided. The instructions used to grade the 
graders are shown in Figure 2. A cardinal and ordinal score are assigned to the graders, similar to the peer grading process of the reports.

\section{Grade the Grader Instructions}

You have been graded by three teams (including you). Provide a cardinal rank of the quality of the grading you received. Consider the following as you assess the grader's grading. Did they give you high marks? Is it clear why? Did they give you low marks? Is it clear why? If you got low marks, by using the graders feedback, do you know how you could have improved your work to get high marks?

Give each grading team a cardinal score on a scale from 4 to zero with 4 being best. Also, rank the grading teams in ordinal fashion from best to worst. Note, the cardinal and ordinal ratings should track.

Best quality of grading ___ cardinal score

Second best quality of grading ___ ; cardinal score

Third best quality of grading ___ ; cardinal score

Figure 2 - Instructions for the grading the grader process

The central purpose of grading the graders is to provide accountability during the peer grading process. For example, if Team A does not provide any feedback justifying the grade they assigned to Team B, then Team B will assign Team A low scores due to poor feedback quality, which negatively affects the overall grade for Team A. Grading the graders also encourages teams to evaluate the feedback provided by others and consider the suggested methods to improve their work.

\subsection{Team evaluation procedure}

Each student is also required to complete private evaluations of their fellow team members and themselves. Using the handout shown in Figure 3, each team member is ranked in ordinal fashion according to the best contribution to the worst (or least) contribution. A cardinal score is also required similar to the peer grading process. A written assessment of each member is required to validate the rankings given.

\section{Team Evaluation Instructions}

Evaluate all project team members including yourself. First, list team mates in order of best to worst (or least best) contribution to the assignment. Do not forget to include/rank yourself. Second, add a brief written statement of the contribution the team member made to the project. Third, include a cardinal score for each member on a scale from 4 to zero with 4 being best. Note, identical cardinal scores may be used for multiple team members. The cardinal and ordinal ratings should track.

\begin{tabular}{|l|l|l|}
\hline Names (in ordinal rank) & Peer Assessment & Cardinal Score (4, 3, 2, 1, or 0) \\
\hline & & \\
\hline & & \\
\hline & & \\
\hline & & \\
\hline
\end{tabular}

Figure 3 - Instructions for team evaluations 
The purpose of team evaluations is to maintain accountability within each team and to motivate equal participation from all members. The feedback received from students on team evaluations is confidential and for instructor use only. The evaluations provide the instructor with information regarding team dynamics which affects the participation grade for each student in the course.

\section{Qualitative survey results}

A survey is designed to verify whether or not peer grading is a useful tool for teaching engineering design and whether or not peer grading enhances general student experiences related to grading, motivation, and teamwork. Thirty-two students complete the survey at the end of the semester regarding the peer grading method. The first 13 statements are addressed using a 5point Likert scale to measure the efficacy of peer grading. The survey also features six openended questions, which are interpreted in Section 4.4. The Likert scale survey results are presented below.

\subsection{Peer grading benefits related to design}

The responses to the first five statements of the survey demonstrate the value of the peer grading method when teaching an engineering design course. The survey results to the first five statements are shown in Figure 4. The majority of students agree that peer grading reinforces and improves their understanding of design concepts presented in lecture. Nearly all students agree that peer grading exposes them to various design examples, and zero responses indicate disagreement with this statement. More than $60 \%$ of students agree that peer grading increases their confidence using the design techniques taught in the course and contributes to their design thinking development. Interestingly, none of the students indicate strong disagreement with any of the first five survey statements.

\section{Peer Grading Benefits Related to Design}

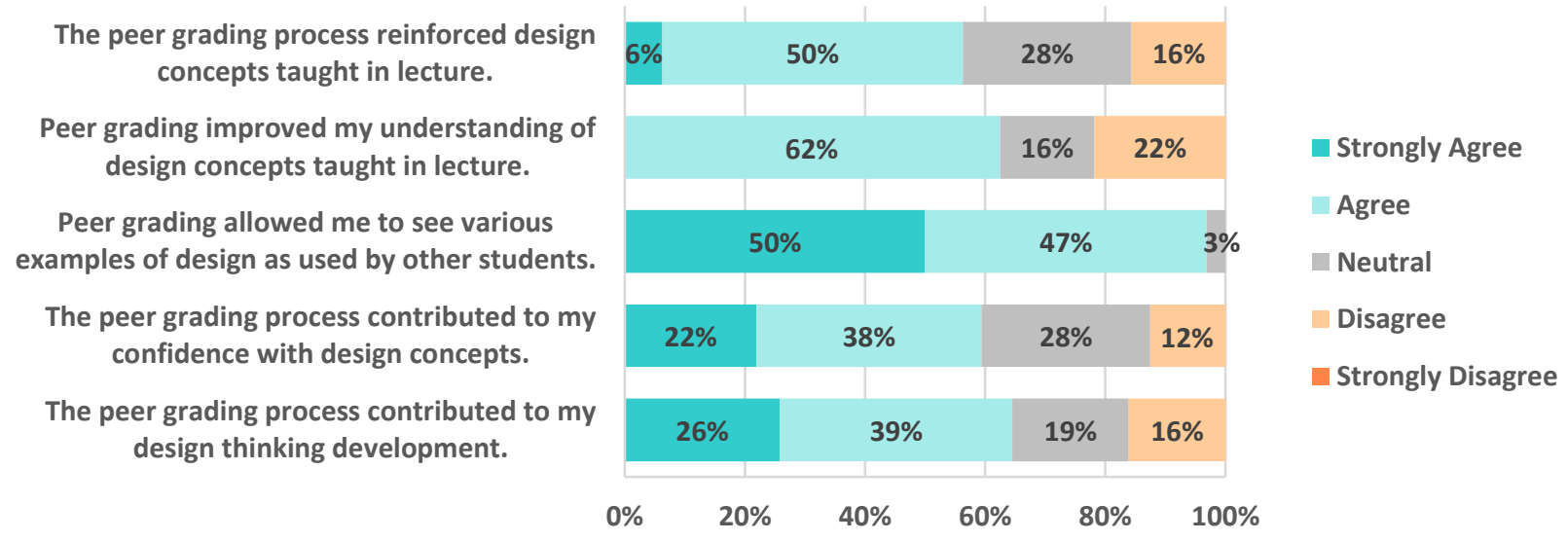

Figure 4 - Comparison of survey responses related to design learning through peer grading 


\subsection{General peer grading benefits}

Five additional statements investigate the benefits of peer grading related to general student learning and achievement. The survey results for these five additional statements are shown in Figure 5. Nearly $70 \%$ of students agree that the peer grading method makes them feel more involved with their grades and personal progress in the course. Peer grading helps approximately $37 \%$ of students feel less anxiety about their progress in the course due to their active participation in the grading process, while the remaining $62 \%$ of the class are neutral or disagree with this statement. The peer grading method motivates roughly $70 \%$ of the class to produce higher quality work considering peers are evaluating their work. Approximately $56 \%$ of students agree that peer grading provides constructive feedback, while $22 \%$ do not agree that they receive constructive feedback through peer grading. Lastly, the majority of students agree that peer grading leads to student participation and provides teamwork opportunities.

\section{General Peer Grading Benefits}

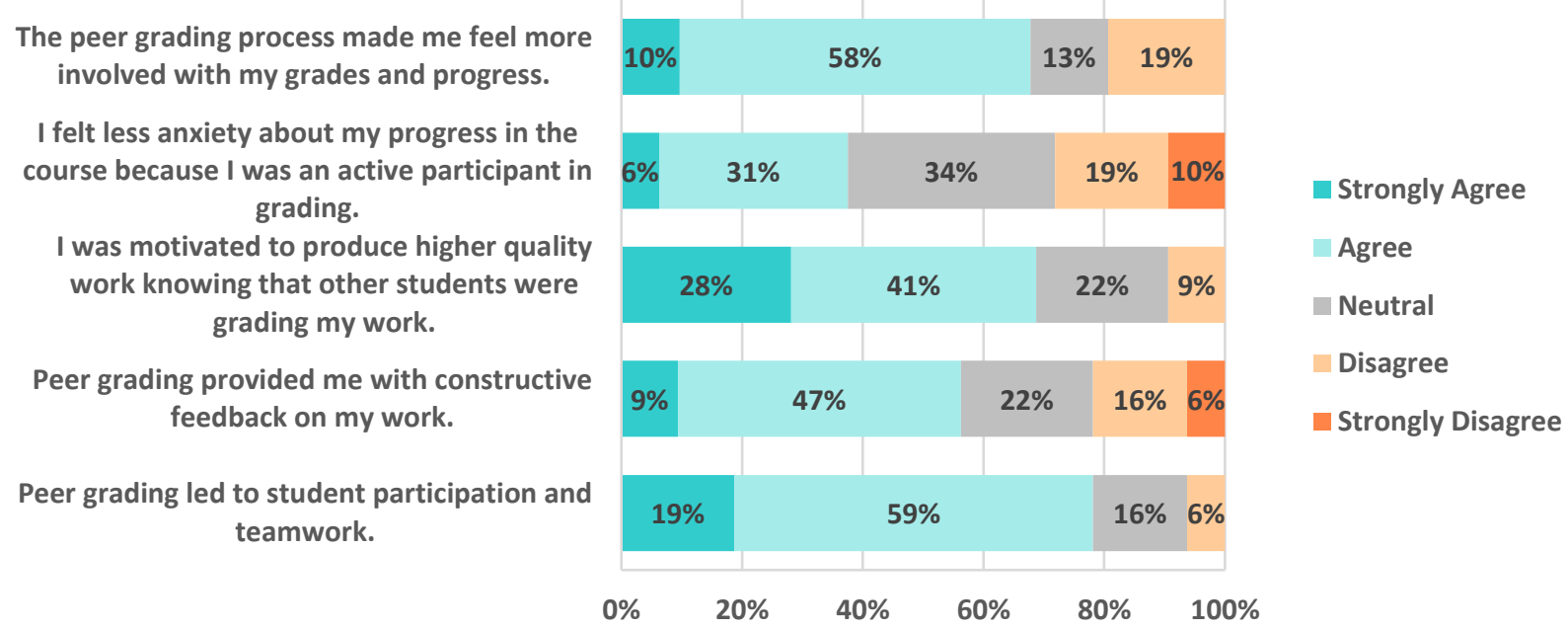

Figure 5 - Comparison of survey responses related to general benefits of peer grading

\subsection{Satisfaction with peer grading method}

The final three survey statements are included in the survey to investigate student satisfaction with the peer grading method. The responses to these three statements are shown in Figure 6. Approximately $56 \%$ of students have a positive view of peer grading, while $25 \%$ of students disagree that their overall view of peer grading is positive. The responses indicate that $44 \%$ of students are able to clearly follow the grading rubric. The responses also indicate that $34 \%$ of students disagree that peer grading fairly assesses their knowledge of course material. 


\section{Peer Grading Satisfaction}

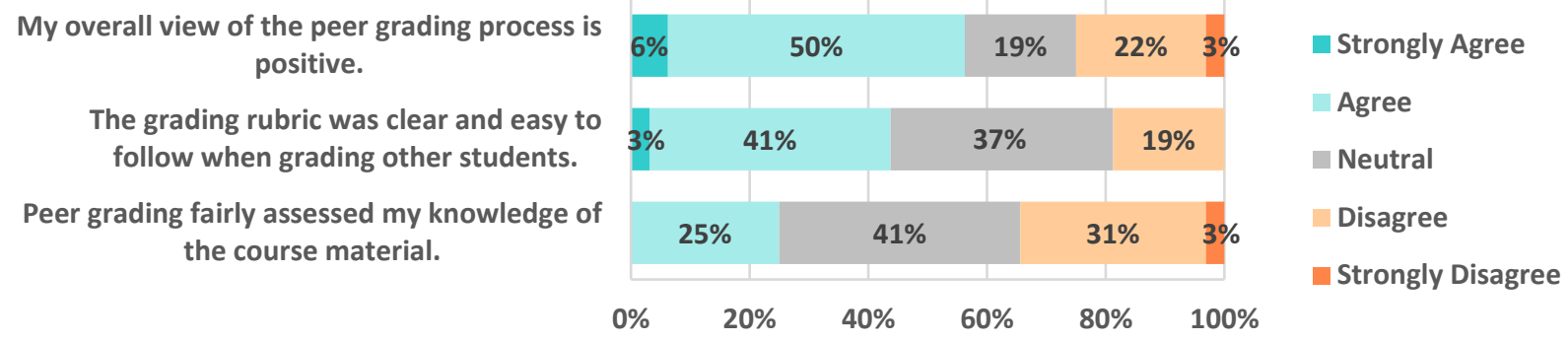

Figure 6 - Comparison of survey responses related to student satisfaction of peer grading

\section{Discussion}

The results presented in Section 3 are discussed further in Sections 4.1-4.3. Where applicable, student responses to the open-ended survey questions are used to provide valuable insight to the Likert scale data shown previously in Figures 4-6. The open-ended survey responses are also discussed in Section 4.4 to provide suggestions for improvement of the peer grading method.

\subsection{Peer grading benefits related to design}

The Likert scale data shown in Figure 4 indicates the peer grading method presented here is a valuable technique for teaching engineering design concepts. Approximately $64 \%$ of students agree that peer grading contributed to their design thinking development. Design thinking refers to "the complex processes of inquiry and learning that designers perform in a systems context, making decisions as they proceed, often working collaboratively on teams in a social process...." [19]. Peer grading requires students to make collaborative decisions about the design processes of themselves and their peers, which improves their understanding and retention of engineering design concepts as supported by the Likert scale data. In this way, peer grading is a useful tool to increase student's understanding of engineering design concepts and to promote design thinking. Nevertheless, a consistent minority of students disagree or are neutral with the first five survey statements related to design, shown in Figure 4. Interestingly, when asked about the usefulness of the peer grading method in an open-ended survey question, the same consistent minority of students explicitly state that while peer grading helps them understand design concepts, they still do not believe peer grading is useful simply because of the poor feedback and unfair grades given by their peers. Therefore, the observation is made that these students allowed irrelevant factors affect their survey responses to the first five statements related to design. To address this issue in the future, students should be made aware beforehand that peer grading is primarily a learning tool, which may shift their focus toward quality of learning rather than focusing on feedback and grades [17]. Considering feedback quality and grading fairness are not the fundamental objectives of peer grading, the authors conclude that the peer grading method successfully reinforces and improves understanding of engineering design concepts taught in lecture. Therefore, the fundamental objective of peer grading is achieved as clearly supported by the survey responses. 


\subsection{General peer grading benefits}

The survey data shown in Figure 5 indicates that the peer grading method helps students feel a greater sense of involvement in their progress, provides teamwork opportunities, and motivates the students to produce higher quality work. However, three to four students express strong disagreement to the following two survey statements: "I felt less anxiety about my progress in the course because I was an active participant in grading" and "Peer grading provided me with constructive feedback on my work." Interestingly, the students who strongly disagree with these two statements express their preference for professor grading over peer grading in the openended survey responses. Therefore, the authors speculate that implementing professor grading after each peer grading exercise may alleviate the strong disagreement to these two statements. Students may feel less anxiety about their progress in the course if they receive the professor's feedback on peer graded assignments. Professor grading may also provide students with greater quality feedback and a sense of confidence in the feedback received from their peers considering it will be reviewed by the professor. The authors believe that the strong disagreement to these two survey statements from three to four students is not an indication that the peer grading method is ineffective as a teaching strategy. Rather, the responses provide insight for improving the peer grading method through the implementation of professor grading after each peer grading exercise.

\subsection{Peer grading satisfaction}

The survey data shown in Figure 6 indicates the majority of students have a positive view of the peer grading process. Only one student strongly disagrees with this survey statement. In an openended survey response, the same student expresses strong opposition to peer grading on the sole basis that students are inexperienced and the professor is the only one who is qualified to properly and consistently grade reports. The authors believe that this strong opposition to the current peer grading method may be alleviated upon implementation of professor grading after each peer grading exercise to ensure fair grading, higher quality peer feedback, and acceptance of the peer feedback received considering it will be reviewed and graded by the professor.

The second survey statement in Figure 6 regarding the clarity of the grading rubric shows mixed results. While $44 \%$ of students agree that they are able to clearly follow the grading rubric, $20 \%$ of students disagree with this statement. Of the students that disagree, the common suggestion in open-ended survey responses for improvement of the peer grading process is a more rigorously defined grading rubric. While a more detailed grading rubric may improve clarity, the authors also believe that proper training is required for future implementation of peer grading to allow the students to practice using the grading rubric and resolve potential discrepancies or misunderstandings that may arise during the training session.

The third survey statement in Figure 6 regarding the fairness of peer grading as an assessment tool also shows mixed results. While $25 \%$ of students agree that peer grading fairly assesses their knowledge of course material, $34 \%$ of students disagree with this statement. Interestingly, all students that disagree with this survey statement express preference for professor grading over peer grading in their open-ended survey responses. This preference may have led them to believe that peer grading is not as fair as professor grading. Some of these students also suggest a 
redistribution of final grade weights so that the peer graded assignments minimally affect their final grade. This suggestion may indicate that students viewed the peer grading method as unfair due to the weighting scheme of peer graded assignments. However, the authors believe that reducing the weight of peer grading assignments may also decrease motivation to do well on peer grading assignments. Though the fundamental objective of peer grading is to reinforce design concepts taught in lecture, and the survey data indicates that the fundamental objective is achieved, the authors acknowledge that improving the perceived fairness of peer grading may be beneficial for future implementation.

\subsection{Interpretation of open-ended survey responses}

The open-ended survey responses provide valuable suggestions for future work that may improve the peer grading method. The primary concerns among students are related to reducing biased, unfair grading and increasing the quality of constructive feedback. The authors believe these concerns may be resolved using the following student suggestions described below.

Several students suggest that the grading rubric is not clear and that discrepancies arise due to a lack of training using the rubric, such as variances in interpretation of scoring. Research shows that providing students with comprehensive training before implementing peer grading is beneficial $[14,17,20]$. Merely providing the students with a grading rubric may lead to confusion and scoring discrepancies. Therefore, training the students to use the rubric on practice assignments is essential for future implementation.

Several students indicate that others grade based on personal bias rather than following the grading rubric, which results in poor quality feedback and unfair grades. Students also express confusion regarding certain grading criteria in the rubric which leads to erroneous scoring. A common suggestion from student responses is to revise the peer grading rubric with greater detail and clarity to emphasize the importance of providing high quality feedback and to ensure fair, unbiased grading.

Students suggest reducing the grade weights of the peer grading assignments. Students believe that peer grades should not affect their final grade in the course considering the students are inexperienced graders and allow bias to affect their grading efforts. However, significantly reducing the grade weights of peer graded assignments may also significantly reduce motivation for students to thoroughly assess their peers, thereby defeating the purpose of peer grading. Students also suggest implementing a heavier weight for the grade the grader procedure to enforce a higher level of accountability to graders, which may reduce grading bias and improve feedback quality.

The most common suggestion for improving the peer grading method is to integrate professor grading after peer grading. Students are not confident in the ability of their peers to properly assess their work and are accustomed to receiving feedback from the professor. Therefore, implementing professor grading after peer grading is essential. Receiving constructive feedback from the professor may increase the student's confidence using design techniques taught in lecture. Implementing an additional round of grading by the professor after each peer grading exercise may also provide students with greater quality feedback and less anxiety about their 
progress in the course considering their work is graded by the professor as well as their fellow students. Further, professor grading after peer grading may foster higher accountability among students during peer grading considering the professor will be reviewing their feedback.

Students criticize the peer grading method for various reasons in the open-ended survey responses. Some students question the ability of their peers to effectively grade material considering they are novices in design compared to the professor. While this is a valid concern, the peer grading method is only used on two class assignments. The professor grades all other assignments, provides the final grade, and evaluates peer grading scores with minimal weighting. Few students also complained that peer grading is time-consuming. However, the authors argue that this investment of time is worthwhile considering the peer grading method reinforces and improves understanding of engineering design concepts.

\section{Conclusions and future work}

A peer grading method is developed and integrated into a graduate-level engineering product design course. The fundamental course objective is to equip students with skills necessary for success as a design engineer or design researcher. The peer grading method presented here differs from prior efforts in the literature where peer grading is used in an engineering design course because student teams assess the reports of other teams, which exposes students to more design examples and helps them learn from their peers. The peer grading method presented here is also different from prior efforts in the literature where peer grading is used in an engineering design course because students are required to grade their graders, which provides accountability to graders and encourages students to evaluate and implement peer feedback. A survey is given to students at the end of the semester to qualitatively measure the efficacy of the peer grading method in supporting course objectives. Qualitative analysis indicates the peer grading method reinforces and improves student understanding of design concepts presented in lecture. The survey results show that peer grading also provides teamwork opportunities, increases student motivation to produce higher quality work, and helps students feel more involved with their grades and overall progress. Reducing biased, unfair grading and increasing the quality of constructive feedback are two primary areas of concern regarding peer grading. Proposed methods for directly addressing these areas of concern include training students for peer grading, revising the grading rubric, modifying weight distributions of peer grades, and implementing professor grading of each peer graded assignment. Despite these suggested methods for improvement, the peer grading method presented here is a valuable technique for teaching engineering design concepts.

\section{References}

[1] Torrance, H., 2007, "Assessment as learning? How the use of explicit learning objectives, assessment criteria and feedback in post-secondary education and training can come to dominate learning. 1," Assessment in Education, 14(3), pp. 281-294.

[2] Topping, K., 1998, "Peer assessment between students in colleges and universities," Review of educational Research, 68(3), pp. 249-276.

[3] Yoo, M. S., and Chae, S. M., 2011, "Effects of peer review on communication skills and learning motivation among nursing students," Journal of Nursing Education, 50(4), pp. 230-233. 
[4] Casey, D., Burke, E., Houghton, C., Mee, L., Smith, R., Van Der Putten, D., Bradley, H., and Folan, M., 2011, "Use of peer assessment as a student engagement strategy in nurse education," Nursing \& health sciences, 13(4), pp. 514-520.

[5] Liu, N.-F., and Carless, D., 2006, "Peer feedback: the learning element of peer assessment," Teaching in Higher education, 11(3), pp. 279-290.

[6] Orsmond, P., Merry, S., and Reiling, K., 1996, "The importance of marking criteria in the use of peer assessment," Assessment \& Evaluation in Higher Education, 21(3), pp. 239-250.

[7] Boud, D., and Falchikov, N., 2007, Rethinking assessment in higher education: Learning for the longer term, Routledge.

[8] Morris, E., McAdams, D. A., "A Review of Practical Design Integration Methods for Existing Engineering Curriculum," Proc. 2015 ASEE Annual Conference.

[9] Willey, K., and Gardner, A., 2010, "Investigating the capacity of self and peer assessment activities to engage students and promote learning," European Journal of Engineering Education, 35(4), pp. 429-443.

[10] McKeown, R., Clarke, R., Griffiths, S., Houston, K., and Lazenblatt, A., 1995, "Product development team projects in final year engineering design," Enhancing Student learning through peer tutoring in higher education: Section, pp. 79-86.

[11] Clark, N., Davies, P., and Skeers, R., "Self and peer assessment in software engineering projects," Proc. Proceedings of the 7th Australasian conference on Computing education-Volume 42, Australian Computer Society, Inc., pp. 91-100.

[12] Rafiq, Y., and Fullerton, H., 1996, "Peer assessment of group projects in civil engineering," Assessment and Evaluation in Higher Education, 21(1), pp. 69-81.

[13] Gehringer, E. F., 2001, "Electronic peer review and peer grading in computer-science courses," ACM SIGCSE Bulletin, 33(1), pp. 139-143.

[14] Cheng, W., and Warren, M., 1997, "Having second thoughts: student perceptions before and after a peer assessment exercise," Studies in Higher Education, 22(2), pp. 233-239.

[15] Sadler, P. M., and Good, E., 2006, "The impact of self-and peer-grading on student learning," Educational assessment, 11(1), pp. 1-31.

[16] Freeman, S., and Parks, J. W., 2010, "How accurate is peer grading?," CBE-Life Sciences Education, 9(4), pp. 482-488.

[17] Dochy, F., Segers, M., and Sluijsmans, D., 1999, "The use of self-, peer and co-assessment in higher education: A review," Studies in Higher education, 24(3), pp. 331-350.

[18] Lu, Y., Warren, J., Jermaine, C., Chaudhuri, S., and Rixner, S., "Grading the graders: Motivating peer graders in a MOOC," Proc. Proceedings of the 24th International Conference on World Wide Web, ACM, pp. 680-690.

[19] Dym, C. L., Agogino, A. M., Eris, O., Frey, D. D., and Leifer, L. J., 2005, "Engineering design thinking, teaching, and learning," Journal of Engineering Education, 94(1), pp. 103-120. [20] Horgan, D., Bol, L., and Hacker, D., 1997, "An examination of the relationships among self, peer, and instructor assessments," European Association for Research on Learning and Instruction, Athens, Greece. 